\title{
SOME REMARKS AND CORRECTIONS TO ONE OF MY PAPERS
}

\author{
PAUL ERDÖS
}

Professor Hartmann pointed out two inaccuracies in my paper Some remarks about additive and multiplicative functions (Bull. Amer. Math. Soc. vol. 52 (1946) pp. 527-537) (see Mathematical Reviews vol. 7 (1946) p. 577).

His first objection is that my proof of Theorem 12 (see p. 535) assumes that $f\left(p^{\alpha}\right) \geqq 0$. The only place the error occurs is in the fifth formula line of p. 536. But the error is quite easy to correct, only a $O(1)$ term is missing. The correct version of the formula is

$$
\sum_{m=1}^{n} g_{k}(m) \leqq n \sum_{d} \frac{h_{k}(d)}{d}+O(1)<n \prod_{p}\left(1+\frac{h_{k}(p)}{p}\right)+O(1) .
$$

Otherwise the proof is unchanged.

His second objection is against Theorem 13 (pp. 536-537) and is more serious.

Theorem 13 was stated as follows: Let $g(n) \geqq 0$ be multiplicative. Then the necessary and sufficient condition for the existence of the distribution function is that

$$
\sum_{p} \frac{(g(p)-1)^{\prime}}{p}<\infty, \quad \sum_{p} \frac{\left((g(p)-1)^{\prime}\right)^{2}}{p}<\infty
$$

where $(g(p)-1)^{\prime}=g(p)-1$ if $|g(p)-1| \leqq 1$ and 1 otherwise.

I try to prove this by putting $\log g(n)=f(n)$ and state that $g(n)$ has a distribution function if and only if $f(n)$ has a distribution function.

In his review Hartmann points out that first of all this implies $g(n)>0$ (instead of $g(n) \geqq 0$ ), and in a letter he points out that my statement is incorrect if $g(n)$ has a distribution function but $\lim _{x \rightarrow+0} G(x)>0(G(x)$ being the distribution function of $g(x))$. (I seem to remember that in my mind I was somehow unwilling to admit these $G(x)$ as distribution functions, but neglected to state this.)

In fact it is easy to see that this case can occur. Put $g\left(p^{\alpha}\right)=1 / 2$ for all $p$ and $\alpha$. Then $G(x)=1$ for all $x \geqq 0$, but clearly $f(n)$ has no distribution function, and the series (1) do not converge. Thus Theorem 13 is incorrect as it stands. The correct version may be stated as follows:

Received by the editors January 20, 1947. 
Theorem $13^{\prime}$. Let $g(n) \geqq 0$ be multiplicative. Assume that the series (1) converge. Then $g(n)$ has a distribution function. The converse is also true unless $G(x)=1$ for all $x \geqq 0$.

First of all we remark that if

$$
\sum_{0(p)=0} \frac{1}{p}=\infty
$$

we have $G(x)=1$ for all $x \geqq 0$ (since almost all integers are divisible by a $p$ with $g(p)=0)$. Thus this case can be neglected, and we can assume that the primes with $g(p)=0$ can be neglected, since they do not influence the convergence of the series (1) or the existence of the distribution function. ${ }^{1}$

The first part of Theorem 13 follows as on p. 537 of my paper.

Next we investigate the converse. If we assume that $\lim _{x \rightarrow+0} G(x)=0$ the convergence of (1) follows as on p. 537, since in this case it really is true that $g(n)$ has a distribution function if and only if $f(n)$ has a distribution function.

Assume now

$$
\lim _{x \rightarrow+0} G(x)=c>0 .
$$

We shall show $c=1$. Suppose that $c<1$, we shall show that this leads to a contradiction.

Denote by $F(x)$ the density of integers with $f(n)<x$ (where $f(n)$ $=\log g(n))$. Clearly $F(x)$ exists and satisfies $(G(x)$ is a distribution function)

$$
\lim _{x \rightarrow-\infty} F(x)=c>0, \quad \lim _{x \rightarrow+\infty} F(x)=1 \quad(c<1) .
$$

From now on we make constant use of my joint paper with Wintner ${ }^{1}$ (referred to as E.W.). It follows from (3) that there exist real numbers $a$ and $b$ such that

$$
-\infty<a<b<\infty \text { and } F(b)-F(a)>0 .
$$

From (4) and E.W. \$9, p. 717 it follows that $|f(p)|<A$ (except for a sequence of primes $q$ with $\sum 1 / q<\infty$, which can be neglected).

Next we deduce (E.W. $\S 3$, pp. 714-715) that

$$
\sum_{p} \frac{(f(p))^{2}}{p}<\infty \text {. }
$$

1 Amer. J. Math. vol. 61 (1939) pp. 713-721. 
Further it follows that (E.W. $\$ 4$, p. 714)

$$
\left|\sum_{p<x} \frac{f(p)}{p}\right|<B \quad(B \text { independent of } x) .
$$

In $\$ 6$, p. 716 it is shown that from $|f(p)|<A,(4)$ and (5) it follows that

$$
\sum_{m=1}^{n}(f(m))^{2}<C n
$$

But clearly (7) contradicts (3) (since (3) implies that the density of integers with $f(m)>D$ is not less than $c$ for every $D$ ), which completes the proof of Theorem $13^{\prime}$.

The following question can be raised: Let $f(n)$ be additive and assume that for some $a<b$ the density of the integers satisfying $a \leqq f(n) \leqq b$ exists and is different from 0 . Does it then follow that $f(n)$ has a distribution function?

By the same methods as just used we can show that

$$
|f(p)|<c, \quad \sum_{p} \frac{\left(f(p)^{\prime}\right)^{2}}{p}<\infty, \quad \sum_{p} \frac{f(p)^{\prime}}{p}<\infty .
$$

But at present I cannot decide whether the distribution function has to exist.

Professor Hartmann also pointed out the following misprints in my previous paper:

(1) The first sentence of Theorem 12 should read "Let $f\left(p^{\alpha}\right) \leqq C$."

(2) The inequality symbol in the two formula lines at the bottom of p. 535 should be " $\leqq$ " instead of " $>$."

(3) On p. 537, in the line following the third formula line " $(\log g(p))^{1}>\ldots$ " should be " $(\log g(p))^{2}>\ldots$ "

(4) On p. 537, the fifth formula line should be " $\sum(1 / p) \ldots$ " instead of " $\sum(1 / 2) \cdots$. ."

(5) In the next to the last line of the paper, p. $537, " \ldots f(n)$ " should be " ...g(n)."

(6) The first formula on p. 529 should read " . . $\exp \exp (d \phi(n))$ " instead of " . . $\exp \exp (\phi(n))$."

Syracuse University 